\title{
Symplectic fillings and positive scalar curvature
}

\author{
PAOLO LISCA \\ Dipartimento di Matematica \\ Università di Pisa \\ I-56127 Pisa, ITALY
}

Email: lisca@dm.unipi.it

\begin{abstract}
Let $X$ be a $4-$ manifold with contact boundary. We prove that the monopole invariants of $X$ introduced by Kronheimer and Mrowka vanish under the following assumptions: (i) a connected component of the boundary of $X$ carries a metric with positive scalar curvature and (ii) either $b_{2}^{+}(X)>0$ or the boundary of $X$ is disconnected. As an application we show that the Poincaré homology 3 -sphere, oriented as the boundary of the positive $E_{8}$ plumbing, does not carry symplectically semi-fillable contact structures. This proves, in particular, a conjecture of Gompf, and provides the first example of a 3-manifold which is not symplectically semi-fillable. Using work of Frøyshov, we also prove a result constraining the topology of symplectic fillings of rational homology 3-spheres having positive scalar curvature metrics.
\end{abstract}

\section{AMS Classification numbers Primary: 53C15}

Secondary: 57M50, 57R57

Keywords: Contact structures, monopole equations, Seiberg-Witten equations, positive scalar curvature, symplectic fillings

Proposed: Dieter Kotschick

Seconded: Tomasz Mrowka, John Morgan
Received: 27 February 1998

Accepted: 9 July 1998

\section{Copyright Geometry and Topology}




\section{Introduction}

\subsection{Basic facts and questions on contact structures}

Let $Y$ be a closed 3 -manifold. A coorientable field of 2 -planes $\xi \subset T Y$ is a contact structure if it is the kernel of a smooth 1 -form $\theta$ on $Y$ such that $\theta \wedge d \theta \neq 0$ at every point of $Y^{1}$. Notice that since $\xi$ is oriented by the restriction of $d \theta$ the manifold $Y$ is necessarily orientable. Moreover, an orientation on $Y$ induces a coorientation on $\xi$ and vice-versa. When $Y$ has a prescribed orientation, $\xi$ is said to be positive (negative, respectively), if the orientation on $Y$ induced by $\xi$ coincides with (is the opposite of, respectively) the given one. In this paper we shall only consider oriented 3-manifolds. Therefore, from now on by the expression "3-manifold" we shall always mean "oriented 3-manifold", and all contact structures will be implicitly assumed to be positive.

By the work of Martinet and Lutz [21] we know that every closed, oriented 3-manifold $Y$ admits a positive contact structure. Eliashberg defined a special class of contact structures, which he called overtwisted, and proved that in any homotopy class of cooriented 2-plane fields on a 3 -manifold there exists a unique positive overtwisted contact structure up to isotopy [5]. Eliashberg called tight the non-overtwisted contact structures. For tight contact structures, the questions of existence and uniqueness in a given homotopy class have a negative answer, in general. For instance, Bennequin proved that there exist homotopic, non-isomorphic contact structures on $S^{3}$ [2], while Eliashberg showed that the set of Euler classes of tight contact structures (considered as oriented 2-plane bundles) on a given 3-manifold is finite [7].

The only tight contact structures known at present are fillable in one sense or another, ie, loosely speaking, they are a 3 -dimensional phenomenon induced by a 4-dimensional one. There exist several different notions of fillability for a contact structure, but here we shall only define two of them (the weakest ones). The reader interested in a comprehensive account can look at the survey [12].

A 4-manifold with contact boundary is a pair $(X, \xi)$, where $X$ is a connected, oriented smooth 4-manifold with boundary and $\xi$ is a contact structure on $\partial X$ (positive with respect to the boundary orientation). A compatible symplectic form on $(X, \xi)$ is a symplectic form $\omega$ on $X$ such that $\left.\omega\right|_{\xi}>0$ at every point of $\partial X$. A contact $3-$ manifold $(Y, \zeta)$ is called symplectically fillable if there exists a 4 -manifold with contact boundary $(X, \xi)$ carrying a compatible symplectic

\footnotetext{
${ }^{1}$ For an introduction to contact structures and a guide to the literature we refer the reader to $[2,7,14]$
} 
form $\omega$ and an orientation-preserving diffeomorphism $\phi$ from $Y$ to $\partial X$ such that $\phi_{*}(\zeta)=\xi$. The triple $(X, \xi, \omega)$ is said to be a symplectic filling of $Y$. More generally, $(Y, \zeta)$ is called symplectically semi-fillable if the diffeomorphism $\phi$ sends $Y$ onto a connected component of $\partial X$. In this case $(X, \xi, \omega)$ is called a symplectic semi-filling of $Y$. If $(Y, \zeta)$ is symplectically semi-fillable, then $\zeta$ is tight by a theorem of Eliashberg and Gromov (see $[6,19]$ ).

One of the aims of this paper is to address a fundamental question about the fillability of contact 3-manifolds (cf [7], question 8.2.1, and [16], question 4.142):

Question 1.1 Does every oriented 3-manifold admit a fillable contact structure?

Eliashberg's Legendrian surgery construction $[5,15]$ provides a rich source of contact 3-manifolds which are filled by Stein surfaces (a special kind of 4manifolds with contact boundary carrying exact compatible symplectic forms). Symplectically fillable contact structures are not necessarily fillable by Stein surfaces. For example, the 3 -torus $S^{1} \times S^{1} \times S^{1}$ carries infinitely many isomorphism classes of symplectically fillable contact structures, but Eliashberg showed [8] that only one of them can be filled by a Stein surface.

Gompf studied systematically the fillability of Seifert 3-manifolds using Eliashberg's construction. This led him to formulate the following:

Conjecture 1.2 ([15]) The Poincaré homology sphere, oriented as the boundary of the positive $E_{8}$ plumbing, does not admit positive contact structures which are fillable by a Stein surface.

Another basic question asks about the uniqueness of symplectic fillings. Via Legendrian surgery one can construct, for instance, non-diffeomorphic (even after blow-up) symplectic fillings of a given 3-manifold. On the other hand, $S^{3}$ is known to have just one symplectic filling up to blow-ups and diffeomorphisms [6]. We may loosely formulate the uniqueness question as follows (cf question 10.2 in [6] and question 6 in [12]):

Question 1.3 To what extent does a 3-manifold determine its symplectic fillings? 


\subsection{Statement of results}

Some progress in the understanding of contact structures has recently come from studying the spaces of solutions to the Seiberg-Witten equations. One of the outcomes of [20] was a proof of the existence, for every natural number $n$, of homology 3-spheres carrying more than $n$ homotopic, non-isomorphic tight contact structures. Generalizing to a non-compact setting the results of $[25,26]$, Kronheimer and Mrowka [17] introduced monopole invariants for smooth 4manifolds with contact boundary, and used them to strengthen the results of [20] as well as to prove new results, as for example that on every oriented 3-manifold there is only a finite number of homotopy classes of symplectically semi-fillable contact structures. In this paper we apply [17] to establish the following:

Theorem 1.4 Let $(X, \xi)$ be a 4-manifold with contact boundary equipped with a compatible symplectic form. Suppose that a connected component of the boundary of $X$ admits a metric with positive scalar curvature. Then, the boundary of $X$ is connected and $b_{2}^{+}(X)=0$.

The following corollary of theorem 1.4 proves conjecture 1.2 as a particular case, and provides a negative answer to question 1.1.

Corollary 1.5 Let $Y$ denote the Poincaré homology sphere oriented as the boundary of the positive $E_{8}$ plumbing. Then, $Y$ has no symplectically semifillable contact structures. Moreover, $Y \#-Y$ is not symplectically semi-fillable with any choice of orientation.

Proof Since $Y$ is the quotient of $S^{3}$ by a finite group of isometries acting freely, it has a metric with positive scalar curvature. Hence, by theorem 1.4 if $Y$ is symplectically semi-fillable then it is symplectically fillable. Moreover, observe that $Y$ cannot be the oriented boundary of a smooth oriented and negative definite $4-$ manifold. In fact, if $\partial X=Y$ then $X \cup\left(-E_{8}\right)$ is a closed, smooth oriented 4-manifold with a definite and non-standard intersection form. The existence of such a 4-manifold is forbidden by the well-known theorem of Donaldson [3, 4]. In view of theorem 1.4, this proves the first part of the statement. The second part follows from a general result of Eliashberg: if $M \# N$ is symplectically semi-fillable, then both $M$ and $N$ are (see [6], theorem 8.1).

Theorem 1.4 can be used, in conjunction with [13], to address question 1.3. Let $(X, \xi)$ be a 4 -manifold with contact boundary equipped with a compatible 
symplectic form. Let $Q_{X}: H_{2}(X ; \mathbb{Z}) /$ Tor $\rightarrow \mathbb{Z}$ be the intersection form of $X$. Write the intersection lattice $J_{X}=\left(H_{2}(X ; \mathbb{Z}) /\right.$ Tor, $\left.Q_{X}\right)$ as

$$
J_{X}=m(-1) \oplus \widetilde{J_{X}}
$$

for some $m$, where $\widetilde{J}_{X}$ does not contain classes of square -1 .

Corollary 1.6 Let $Y$ be a rational homology sphere having a positive scalar curvature metric. Then, while $X$ ranges over the set of symplectic fillings of $Y$ such that $\widetilde{J}_{X}$ is even, the set of isomorphism classes of the lattices $\widetilde{J}_{X}$ ranges over a finite set.

Proof By a result of Frøyshov ([13], theorem 1) there exists a rational number $\gamma(Y) \in \mathbb{Q}$ depending only on $Y$ such that if $X$ is a negative 4 -manifold bounding $Y$, then for every characteristic element $\xi \in H_{2}(X, \partial X ; \mathbb{Z}) /$ Tor (ie such that $\xi \cdot x \equiv x \cdot x \bmod 2$ for every $x \in H_{2}(X, \mathbb{Z}) /$ Tor $)$, the following inequality holds:

$$
\operatorname{rank}\left(J_{X}\right)-|\xi|^{2} \leq \gamma(Y)
$$

Thus, if $X$ is a symplectic filling of $Y$, by theorem $1.4 b_{2}^{+}(X)=0$ and therefore equation (1.1) holds. Clearly (1.1) is also true with $\widetilde{J}_{X}$ in place of $J_{X}$. Hence, if $\widetilde{J}_{X}$ is even, choosing $\xi=0$ we see that the rank of $\widetilde{J}_{X}$ is bounded above by a constant depending only on $Y$. On the other hand, the absolute value of its determinant is bounded above by the order of $H_{1}(Y ; \mathbb{Z})$. It follows (see eg [22]) that the isomorphism class of $\widetilde{J}_{X}$ must belong to a finite set determined by $Y$.

Remark 1.7 The conclusion of corollary 1.6 can be strengthened in particular cases. For example, if $Y$ is an integral homology sphere, then the intersection lattice $J_{X}$ of any symplectic filling of $Y$ is unimodular. It follows from $[9,10]$ that if $\gamma(Y) \leq 8$ then, regardless of whether $\widetilde{J}_{X}$ is even or odd, there are exactly 14 (explicitly known) possibilities for the isomorphism class of $\widetilde{J}_{X}$ (due to recent work of Mark Gaulter this is still true as long as $\gamma(Y) \leq 24$ [11]). In particular, if $Y$ is the Poincaré 3 -sphere oriented as the boundary of the negative plumbing $-E_{8}$, then $\gamma(Y)=8$ [13]. Up to isomorphism the only even, negative and unimodular lattices of rank at most eight are 0 and $-E_{8}$. Therefore, 0 and $-E_{8}$ are the only possibilities for $\widetilde{J}_{X}$ in this case. Moreover, notice that if $Y$ bounds a smooth 4 -manifold with $b_{2}=0$, the same is true for $-Y$. On the other hand, the argument given to prove corollary 1.5 shows that $-Y$ cannot bound negative semi-definite manifolds. Therefore, if $X$ is an even symplectic filling of $Y, J_{X}$ is necessarily isomorphic to the negative lattice $-E_{8}$. 
In view of corollary 1.6 and remark 1.7 it seems natural to formulate the following conjecture:

Conjecture 1.8 The conclusion of corollary 1.6 still holds, under the same assumptions, if $X$ is allowed to range over the set of all symplectic fillings of $Y$.

The plan of the paper is the following. In section 2 we initially fix our notation recalling the results of [17]. Then we state and prove, for later reference, an immediate consequence of those results, observing how it implies a theorem of Eliashberg. In section 3 we prove our main result, theorem 3.2, and its corollary theorem 1.4. The line of the argument to prove theorem 3.2 is well-known to the experts. It is the analogue, in the context of 4 -manifolds with contact boundary, of a standard argument proving the vanishing of the Seiberg-Witten invariants of a closed smooth 4 -manifold which splits as a union $X_{1} \bigcup_{Y} X_{2}$, with $Y$ carrying a positive scalar curvature metric and $b_{2}^{+}\left(X_{i}\right)>0, i=1,2$ (cf [18], remark 6). The crucial points of such an argument depend on the technical results of [23].

Acknowledgements. It is a pleasure to thank Dieter Kotschick for his interest in this paper, and for useful comments on a preliminary version of it. Warm thanks also go to Peter Kronheimer for observing that the assumption $b_{2}^{+}>0$ in theorem 3.2 could be disposed of when the boundary is disconnected, and to Yasha Eliashberg for pointing out the second part of corollary 1.5. Finally, I am grateful to the referee for her/his remarks.

\section{Preliminaries}

We start describing the set-up of [17] (the reader is referred to the original paper for details). A $\operatorname{Spin}^{c}$ structure on a smooth 4 -manifold $X$ is a triple $\left(W^{+}, W^{-}, \rho\right)$, where $W^{+}$and $W^{-}$are hermitian rank-2 bundles over $X$ called respectively the positive and negative spinor bundle, and $\rho: T^{*} X \rightarrow$ $\operatorname{Hom}\left(W^{+}, W^{-}\right)$is a linear map satisfying the Clifford relation: $\rho(\theta)^{*} \rho(\theta)=$ $|\theta|^{2} \operatorname{Id}_{W^{+}}$for every $\theta \in T^{*} X$. The map $\rho$ extends to a linear embedding $\rho: \Lambda^{*} T^{*} X \rightarrow \operatorname{Hom}\left(W^{+}, W^{-}\right)$. A Spin connection $A$ is a unitary connection on $W=W^{+} \oplus W^{-}$such that the induced connection on $\operatorname{End}(W)$ agrees with the Levi-Civita connection on the image of $\rho$. To any Spin connection $A$ is associated, via $\rho$, a twisted Dirac operator $D_{A}^{+}: \Gamma\left(W^{+}\right) \rightarrow \Gamma\left(W^{-}\right)$. 
Given a $4-$ manifold with contact boundary $(X, \xi)$, let $X^{+}$be the smooth manifold obtained from $X$ by attaching the open cylinder $[1,+\infty) \times \partial X$ along $\partial X$. Up to certain choices, the contact structure $\xi$ determines on $[1,+\infty) \times \partial X$ a metric $g_{0}$ and a self-dual 2 -form $\omega_{0}$ of constant length $\sqrt{2}$. $\omega_{0}$ determines on $[1,+\infty) \times \partial X$ a $\operatorname{Spin}^{c}$ structure $\mathbf{s}_{0}=\left(W^{+}, W^{-}, \rho\right)$ and a unit section $\Phi_{0}$ of $W^{+}$. Moreover, there is a unique Spin connection $A_{0}$ such that $D_{A_{0}}^{+}\left(\Phi_{0}\right)=0$. Given an arbitrary extension of $g_{0}$ to all of $X^{+}$, the triple $\left(X^{+}, \omega_{0}, g_{0}\right)$ is an AFAK (asymptotically flat almost Kähler) manifold, in the terminology of [17]. Consider the set $\operatorname{Spin}^{c}(X, \xi)$ of isomorphism classes of $\operatorname{Spin}^{c}$ structures on $X^{+}$ whose restriction to $[1,+\infty) \times \partial X$ is isomorphic to $\mathbf{s}_{0}$. We shall now describe how Kronheimer and Mrowka define a map

$$
\mathrm{SW}_{(X, \xi)}: \operatorname{Spin}^{c}(X, \xi) \rightarrow \mathbb{Z}
$$

which is an invariant of the pair $(X, \xi)$. Given $\mathbf{s}=\left(W^{+}, W^{-}, \rho\right) \in \operatorname{Spin}^{c}(X, \xi)$, extend $\Phi_{0}$ and $A_{0}$ arbitrarily to all of $X^{+}$. Let $L_{l}^{2}$ and $L_{l, A_{0}}^{2}, l \geq 4$ be, respectively, the standard Sobolev spaces of imaginary 1 -forms and sections of $W^{+}$, and let $\mathcal{C}$ be the space of pairs $(A, \Phi)$ such that $A-A_{0} \in L_{l}^{2}$ and $\Phi-\Phi_{0} \in L_{l, A_{0}}^{2}$. Then, $\mathcal{G}=\left\{u: X^{+} \rightarrow \mathbb{C}|| u \mid=1,1-u \in L_{l+1}^{2}\right\}$ is a Hilbert Lie group acting freely on $\mathcal{C}$. Let $\eta \in L_{l-1}^{2}\left(i \mathfrak{s u}\left(W^{+}\right)\right)$. Given a Spin connection $A$, let $\hat{A}$ be the induced $U(1)$ connection on $\operatorname{det}\left(W^{+}\right)$. Let $M_{\eta}(\mathbf{s})$ be the quotient, under the action of $\mathcal{G}$, of the set of pairs $(A, \Phi) \in \mathcal{C}$ which satisfy the $\eta$-perturbed Seiberg-Witten (or monopole) equations

$$
\left\{\begin{array}{l}
\rho\left(F_{\hat{A}}^{+}\right)-\left\{\Phi \otimes \Phi^{*}\right\}=\rho\left(F_{\hat{A}_{0}}^{+}\right)-\left\{\Phi_{0} \otimes \Phi_{0}^{*}\right\}+\eta \\
D_{A}^{+}(\Phi)=0,
\end{array}\right.
$$

where $\left\{\Phi \otimes \Phi^{*}\right\}$ denotes the traceless part of the endomorphism $\Phi \otimes \Phi^{*}$. Kronheimer and Mrowka [17] prove that, for $\eta$ in a Baire set of perturbing terms exponentially decaying along the end, $M_{\eta}(\mathbf{s})$ is (if non-empty) a smooth, compact orientable manifold of dimension $d(\mathbf{s})$ equal to $\left\langle e\left(W^{+}, \Phi_{0}\right),[X, \partial X]\right\rangle$, the obstruction to extending $\Phi_{0}$ as a nowhere-vanishing section of $W^{+}$. Now suppose that an orientation for $M_{\eta}(\mathbf{s})$ has been chosen. Then, when $d(\mathbf{s})=0$ one can define an integer as the number of points of $M_{\eta}(\mathbf{s})$ counted with signs. $S W_{(X, \xi)}(\mathbf{s})$ is defined to be this integer when $d(\mathbf{s})=0$, and zero when $d(\mathbf{s}) \neq 0$.

If $(X, \xi)$ is equipped with a compatible symplectic form $\omega$, then a theorem from [17] says that there are natural choices of an element $\mathbf{s}_{\omega} \in \operatorname{Spin}^{c}(X, \xi)$ and of an orientation of $M_{\eta}\left(\mathbf{s}_{\omega}\right)$ so that $\mathrm{SW}_{(X, \xi)}\left(\mathbf{s}_{\omega}\right)=1$.

The following proposition is implicitly contained in [13] and [17]. Here we give an explicit statement and proof for the sake of clarity and later reference. 
Proposition 2.1 Let $(X, \xi)$ be a 4-manifold with contact boundary. Suppose that $S W_{(X, \xi)}(\mathbf{s}) \neq 0$ for some $\mathbf{s} \in \operatorname{Spin}^{c}(X, \xi)$. If a connected component $Y$ of the boundary of $X$ has a metric with positive scalar curvature then the map $H^{2}(X ; \mathbb{R}) \rightarrow H^{2}(Y ; \mathbb{R})$ induced by the inclusion $Y \subset X$ is the zero map.

Proof The contact structure $\xi$ induces a $\operatorname{Spin}^{c}$ structure $\mathbf{t}$ on $Y$ (see [17]). Let $W$ be the associated spinor bundle on $Y$. Given a closed 2-form $\mu$ on $Y$, denote by $N_{\mu}(Y, \mathbf{t})$ the set of gauge equivalence classes of solutions to the 3-dimensional monopole equations on $Y$ corresponding to the $\operatorname{Spin}^{c}$ structure $\mathbf{t}$ and perturbation $\mu$. As observed in [17], proposition 5.3, it follows from the Weitzenböck formulae and [13] that if $\mu_{0} \in \Omega^{2}(Y)$ is a closed 2-form with $\left[\mu_{0}\right] \neq 2 \pi c_{1}(W)$, then there exists a Baire set of exact $C^{r}$ forms $\mu_{1}$ such that $N_{\mu_{0}+\mu_{1}}(Y, \mathbf{t})$ consists of finitely many non-degenerate, irreducible solutions. Arguing by contradiction, suppose that the restriction map $H^{2}(X ; \mathbb{R}) \rightarrow H^{2}(Y ; \mathbb{R})$ is non-zero. Then, for every real number $\epsilon>0$ there exists a closed 2 -form $\mu$ on $Y$ such that:

(1) $N_{\mu}(Y, \mathbf{t})$ consists of finitely many non-degenerate, irreducible solutions.

(2) the $L^{2}$ norm of $\mu$ is less than $\epsilon$,

(3) $[\mu] \neq 2 \pi c_{1}(W) \in H^{2}(Y ; \mathbb{R})$ and $[\mu]$ is in the image of the restriction map $H^{2}(X ; \mathbb{R}) \rightarrow H^{2}(Y ; \mathbb{R})$.

Since $\mathrm{SW}_{(X, \xi)}(\mathbf{s}) \neq 0$, by [17], proposition $5.8, N_{\mu}(Y, \mathbf{t})$ is non-empty. But since $Y$ has a metric of positive scalar curvature, if $\epsilon$ is sufficiently small the Weitzenböck formulae imply that $N_{\mu}(Y, \mathbf{t})$ is empty: a contradiction.

It is interesting to observe that proposition 2.1 has the following corollary, which was first proved by Eliashberg using the technique of filling by holomorphic disks [5].

Corollary 2.2 $S^{2} \times D^{2}$ has no tame almost complex structure with $J$-convex boundary.

Proof A standard product metric on $S^{2} \times S^{1}$ has positive scalar curvature. Moreover, an almost complex structure on $S^{2} \times D^{2}$ has $J$-convex boundary if, by definition, the distribution $\xi$ of complex tangents to $S^{2} \times S^{1}$ is a positive contact structure. If $J$ is tame, then there is a compatible symplectic form $\omega$ on the 4-manifold with contact boundary $\left(S^{2} \times D^{2}, \xi\right)$. Hence $\mathrm{SW}_{\left(S^{2} \times D^{2}, \xi\right)}\left(\mathbf{s}_{\omega}\right) \neq$ 0 . But the restriction map $H^{2}\left(S^{2} \times D^{2} ; \mathbb{R}\right) \rightarrow H^{2}\left(S^{2} \times S^{1} ; \mathbb{R}\right)$ is non-zero, contradicting proposition 2.1. 


\section{Proofs of the main results}

In this section we prove the main results of the paper, namely theorem 3.2 and its immediate corollary, theorem 1.4. Let $(X, \xi)$ be a 4 -manifold with contact boundary. We shall start with a preliminary discussion under the assumption that the boundary of $X$ is connected and admits a metric with positive scalar curvature. During the proof of theorem 3.2 we will say how to modify the arguments when the boundary of $X$ is possibly disconnected and at least one of its connected components admits a metric with positive scalar curvature.

We begin along the lines of [17], proposition 5.6. Let $\left(X^{+}, g_{0}\right)$ be the Riemannian 4-manifold defined in section 2 . We are going to analyze what happens to the solutions of the equations (2.1) when the metric $g_{0}$ is stretched in the direction normal to the boundary of $X$.

In the following discussion we shall denote the boundary of $X$ by $Y$. Let $g_{Y}$ be a positive scalar curvature metric on $Y$. Let $g_{1}$ be a Riemannian metric on $X^{+}$coinciding with $g_{0}$ on $[1,+\infty) \times Y$ and such that $\left(X^{+}, g_{1}\right)$ contains an isometric copy of the cylinder $[-1,1] \times Y$ with the product metric $d t^{2}+g_{Y}$. Choose a perturbing term $\eta_{1}$ for the monopole equations which vanishes on this cylinder. For every $R \geq 1$ let $g_{R}$ and $\eta_{R}$ be obtained by replacing $[-1,1] \times Y$ with a cylinder isometric to $[-R, R] \times Y$. Denote by $X_{\text {in }}$ and $X_{\text {out }}$, respectively, the compact and non-compact component of the complement of the cylinder in $X^{+}$. Suppose that, for some $\mathbf{s} \in \operatorname{Spin}^{c}(X, \xi), \mathrm{SW}_{(X, \xi)}(\mathbf{s}) \neq 0$. This implies that the moduli space $M_{\eta_{R}}(\mathbf{s})$ is non-empty for all $R$. Since the restriction of $\eta_{R}$ to the cylinder $[-R, R] \times Y$ vanishes, the proof of lemma 5.7 from [17] applies. This says that for every solution $\left[A_{R}, \Phi_{R}\right] \in M_{\eta_{R}}(\mathbf{s})$ the variation of the Chern-Simons-Dirac (CSD for short) functional on the restriction of $\left[A_{R}, \Phi_{R}\right]$ to $[-R, R] \times Y$ is bounded, independent of $R$. Denote by $\widehat{X}_{\text {in }}$ and $\widehat{X}_{\text {out }}$ the Riemannian manifolds obtained by isometrically attaching cylinders $[0, \infty) \times Y$ and $(-\infty, 0] \times \bar{Y}$ with metric $d t^{2}+g_{Y}$ to $X_{\text {in }}$ and $X_{\text {out }}$ respectively, where $\bar{Y}$ denotes $Y$ with the opposite orientation. Let $\eta_{\text {in }}$ and $\eta_{\text {out }}$ on $\widehat{X}_{\text {in }}$ and $\widehat{X}_{\text {out }}$ respectively be compactly supported perturbing terms. Let $R_{i}$ be a sequence going to infinity, and let $\eta_{i}=\eta_{R_{i}}$ be a corresponding sequence of perturbing terms as above converging to $\eta_{\text {in }}$ and $\eta_{\text {out }}$. Since the moduli spaces $M_{\eta_{i}}(\mathbf{s})$ are non-empty for all $i$, up to passing to a subsequence we may assume that there are solutions converging on compact subsets to configurations $\left(A_{\text {in }}, \phi_{\text {in }}\right)$ and $\left(A_{\text {out }}, \phi_{\text {out }}\right)$ on $\widehat{X}_{\text {in }}$ and $\widehat{X}_{\text {out }}$. The configurations $\left(A_{\text {in }}, \phi_{\text {in }}\right)$ and $\left(A_{\text {out }}, \phi_{\text {out }}\right)$ satisfy the monopole equations for $\operatorname{Spin}^{c}$ structures $\mathbf{s}_{\text {in }}$ and $\mathbf{s}_{\text {out }}$, say, with perturbing terms $\eta_{\text {in }}$ and $\eta_{\text {out }}$, and have finite variation of the CSD functional on the cylindrical ends. Denote the moduli spaces of solutions with bounded 
variation of the CSD functional along the end by, respectively, $M_{\eta_{\text {in }}}\left(\widehat{X}_{\text {in }}\right)$ and $M_{\eta_{\text {out }}}\left(\widehat{X}_{\text {out }}, \xi\right)$.

The results of [23] imply that $\left(A_{\text {in }}, \phi_{\text {in }}\right)$, restricted to the slices $\{t\} \times Y$ converges, as $t \rightarrow+\infty$, towards an element of the moduli space $N_{X}(Y)$ of solutions of the unperturbed 3-dimensional monopole equations on $Y$ modulo the gauge transformations which extend over $X$. In other words, there is a map $\partial_{X}: M_{\eta_{\text {in }}}\left(\widehat{X}_{\text {in }}\right) \rightarrow N_{X}(Y)$. For every $\theta \in N_{X}(Y)$, we denote $\partial_{X}^{-1}(\theta)$ by $M_{\eta_{\text {in }}}\left(\widehat{X}_{\text {in }}, \theta\right)$.

Now recall that, since $\operatorname{SW}(X, \xi)(\mathbf{s}) \neq 0$, by the definition of the invariants $d(\mathbf{s})=0$, and the canonical spinor $\Phi_{0}$ can be extended over $X$ to a nowherevanishing section of the bundle $W^{+}$. This is equivalent to saying that $\mathbf{s}$ is the $\operatorname{Spin}^{c}$ structure associated to an almost complex structure $J_{X}$ on $X$ (see [17], lemma 2.1). Let $Z$ be a smooth, oriented Riemannian 4-manifold with boundary $\bar{Y}$ and such that $J_{X}$ extends to an almost complex structure $J_{M}$ on the closed oriented 4 -manifold $M=X \cup_{Y} Z$ (the reason why such a $Z$ exists is explained in eg [15], lemma 4.4; one can always find a $Z$ such that the obstruction to extending $J_{X}$ over $Z$ is concentrated at a finite number of points, and then, in order to kill the obstruction, one can modify $Z$ by connect summing at those points with a suitable number of copies of $S^{2} \times S^{2}$ ). Let $\widehat{Z}$ be the manifold with cylindrical end obtained by attaching $(-\infty, 1] \times \bar{Y}$ to the boundary of $Z$. Fix an extension of $J_{M}$ from $Z$ to $\widehat{Z}$, and call $\mathbf{s}_{\widehat{Z}}$ the $\operatorname{Spin}^{c}$ structure induced on $\widehat{Z}$. Choose an identification of the cylindrical ends of $\widehat{X}_{\text {out }}$ and $\widehat{Z}$ (observe that $\mathbf{s}_{\widehat{Z}}$ is isomorphic to $\mathbf{s}_{\text {out }}$ on the cylindrical end). Also, choose a perturbing term $\eta^{\prime}$ on $\widehat{Z}$ which coincides with $\eta_{\text {out }}$ on the cylindrical end. As before, there is a moduli space $M_{\eta^{\prime}}(\widehat{Z})$, a map $\partial_{X}: M_{\eta^{\prime}}(\widehat{Z}) \rightarrow N_{Z}(\bar{Y})$, and, for every $\theta^{\prime} \in N_{Z}(\bar{Y})$, we denote $\partial_{Z}^{-1}\left(\theta^{\prime}\right)$ by $M_{\eta^{\prime}}\left(\widehat{Z}, \theta^{\prime}\right)$.

Lemma 3.1 For any $\theta_{1} \in N_{X}(Y), \theta_{2} \in N_{Z}(\bar{Y}), M_{\eta_{\text {in }}}\left(\widehat{X}_{\text {in }}, \theta_{1}\right)$ and $M_{\eta^{\prime}}\left(\widehat{Z}, \theta_{2}\right)$ are (possibly empty) smooth manifolds. Moreover, the sum of their expected dimensions equals $-1-b_{1}(Y)$.

Proof By a standard argument (see eg [24]), since the metric $g_{Y}$ has nowhere negative scalar curvature, the moduli space $N_{X}(Y)$ consists of reducible solutions, and the linearization of the equations on $Y$ with appropriate gauge fixing gives a deformation complex whose first cohomology group at a point $[A, 0] \in N_{X}(Y)$ can be identified with $H^{1}(Y ; \mathbb{R}) \oplus \operatorname{ker} D_{A}$. Since $g_{Y}$ has positive scalar curvature, we have $\operatorname{ker} D_{A}=0$ for every $[A, 0] \in N_{X}(Y)$. Moreover, since the dimension of $N_{X}(Y)$ is $b_{1}(Y), N_{X}(Y)$ is smooth, and the Kuranishi 
map from the first to the second cohomology of the deformation complex vanishes. It follows from [23] that every element of $M_{\eta_{\text {in }}}\left(\widehat{X}_{\text {in }}\right)$ converges, along the end, exponentially fast towards an element of $N_{X}(Y)$. This implies that, given any $\theta \in N_{X}(Y), M_{\eta_{\text {in }}}\left(\widehat{X}_{\text {in }}, \theta\right)$ is a (possibly empty) smooth manifold. Exactly the same arguments apply to $M_{\eta^{\prime}}(\widehat{Z})$.

Recall that taking the quotient of $N_{X}(Y)$ by the whole gauge group of $Y$ gives a covering map $p: N_{X}(Y) \rightarrow N(Y)$ with fiber $H^{1}(Y ; \mathbb{Z}) / H^{1}(X ; \mathbb{Z})$. For every $\theta_{1} \in N_{X}(Y)$, denote $p\left(\theta_{1}\right)$ by $\bar{\theta}_{1}$. Let $W_{X}^{+}$be the spinor bundle associated with the $\operatorname{Spin}^{c}$ structure $\mathbf{s}_{\text {in }}$. By [1] and [23] the exponential convergence implies that, given $\theta_{1}=[A, 0]$, the expected dimension of $M_{\eta_{\text {in }}}\left(\widehat{X}_{\text {in }}, \theta_{1}\right)$ is

$$
d_{1}=\frac{1}{4}\left(c_{1}\left(W_{X}^{+}\right)^{2}-2 \chi(X)-3 \sigma(X)\right)-\frac{h^{0}\left(\bar{\theta}_{1}\right)+h^{1}\left(\bar{\theta}_{1}\right)}{2}+\eta_{Y}\left(\bar{\theta}_{1}\right)
$$

where $h^{0}\left(\bar{\theta}_{1}\right)=1$ is the dimension of the stabilizer of the configuration $(A, 0)$, and $h^{1}\left(\bar{\theta}_{1}\right)=b_{1}(Y)$ is the dimension of the first cohomology group of the deformation complex at $(A, 0) \cdot \eta_{Y}\left(\bar{\theta}_{1}\right)$ is the $\eta$-invariant of the relevant boundary operator on $Y$ defining the deformation complex (since we are going to use only well known properties of this operator, we don't need to be more specific, see [24] for more details). Note that the rational number $c_{1}\left(W_{X}^{+}\right)^{2}$ is well defined because by proposition $\left.2.1 c_{1}\left(W_{X}^{+}\right)\right|_{Y}$ is a torsion class.

Similarly, if $\theta_{2} \in N_{Z}(\bar{Y})$, the expected dimension of $M_{\eta^{\prime}}\left(\widehat{Z}, \theta_{2}\right)$ is

$$
d_{2}=\frac{1}{4}\left(c_{1}\left(W_{Z}^{+}\right)^{2}-2 \chi(Z)-3 \sigma(Z)\right)-\frac{h^{0}\left(\bar{\theta}_{2}\right)+h^{1}\left(\bar{\theta}_{2}\right)}{2}+\eta_{\bar{Y}}\left(\bar{\theta}_{2}\right) .
$$

Again, $h^{0}\left(\bar{\theta}_{2}\right)=1$ and $h^{1}\left(\bar{\theta}_{2}\right)=b_{1}(Y)$. Recall that $\eta_{Y}$ changes sign when the orientation of $Y$ is reversed. Moreover, since $h^{0}(\bar{\theta})$ and $h^{1}(\bar{\theta})$ are constant in $\bar{\theta} \in N(Y)$ there is no spectral flow, and therefore $\eta_{Y}(\bar{\theta})$ is constant too. Hence, $\eta_{\bar{Y}}\left(\bar{\theta}_{2}\right)=-\eta_{Y}\left(\bar{\theta}_{2}\right)=-\eta_{Y}\left(\bar{\theta}_{1}\right)$. Finally, observe that the $\operatorname{Spin}^{c}$ structures $\mathbf{s}_{\text {in }}$ and $\mathbf{s}_{Z}$ can be glued together to give a $\operatorname{Spin}^{c}$ structure $\mathbf{s}_{M}$ on the closed manifold $M=X \cup_{Y} Z$. In fact, $\mathbf{s}_{M}$ can be taken to be the $\operatorname{Spin}^{c}$ structure induced by the almost complex structure $J_{M}$ (see the discussion before the statement). It follows that the associated spinor bundle $W_{M}^{+}$satisfies

$$
c_{1}\left(W_{M}^{+}\right)^{2}=2 \chi(M)+3 \sigma(M)
$$

and the formula $d_{1}+d_{2}=-1-b_{1}(Y)$ follows immediately from (3.1) and (3.2).

Theorem 3.2 Let $(X, \xi)$ be a 4-manifold with contact boundary. Suppose that one of the following assumptions holds: 
1) The boundary of $X$ is connected, it admits a metric with positive scalar curvature and $b_{2}^{+}(X)>0$,

2) The boundary of $X$ is disconnected and one of its connected components admits a metric with positive scalar curvature.

Then, the map $\mathrm{SW}_{(X, \xi)}$ is identically zero.

Proof We will start by establishing the conclusion under the first assumption. Arguing by contradiction, suppose that the map $\mathrm{SW}_{(X, \xi)}$ does not vanish. Then, one can argue as in [17], proposition 5.4, and show that, for $\eta_{\text {in }}$ in a Baire set of compactly supported perturbations, if, for some $\theta_{1} \in N_{X}(Y)$, $M_{\eta_{\text {in }}}\left(\widehat{X}_{\text {in }}, \theta_{1}\right)$ is non-empty, then its expected dimension is non-negative (observe that, since the perturbing term is decaying to zero along the cylindrical end, we need $b_{2}^{+}(X)>0$ to rule out reducible solutions). Thus, choosing $\eta_{\text {in }}$ in such a Baire set, the existence of $\left(A_{\text {in }}, \Phi_{\text {in }}\right)$ implies $d_{1} \geq 0$. If we denote by $d_{2}$ the expected dimension of $M_{\eta_{\text {out }}}\left(\widehat{X}_{\text {out }}, \xi, \theta_{2}\right)$ (with the obvious meaning of the symbols), the same argument gives $d_{2} \geq 0$ (no assumption on $b_{2}^{+}$is needed now, because the elements of $M_{\eta_{\text {out }}}\left(\widehat{X}_{\text {out }}, \xi, \theta_{2}\right)$ are asymptotically irreducible on the "conical" end). As explained in [17], subsection 5.4, one can associate to $\theta_{2}$ a homotopy class of 2-plane fields $I\left(\theta_{2}\right)$ on $Y$. As in the proof of proposition 5.6 in [17], the expected dimension of $M_{\eta_{\text {out }}}\left(\widehat{X}_{\text {out }}, \xi, \theta_{2}\right)$ is given by a difference element $\bar{\delta}\left(I\left(\theta_{2}\right), \xi\right.$ ) (see [17], subsection 5.1, for the definition of $\bar{\delta}$; in the case at hand this number is an integer because, by proposition 2.1 , the restriction of $c_{1}\left(W^{+}\right)$to $Y$ is a torsion element). Moreover, $\bar{\delta}\left(I\left(\theta_{2}\right), \xi\right)$ is also equal to the expected dimension of $M_{\eta^{\prime}}\left(\widehat{Z}, \theta_{2}\right)$. This contradicts lemma 3.1. Hence, we have established the conclusion of the theorem under the first assumption.

When the boundary of $X$ is disconnected the above argument can be easily modified so that the requirement on $b_{2}^{+}(X)$ becomes redundant. In fact, one can repeat the same construction involving only the end corresponding to the boundary component having positive scalar curvature. $\widehat{X}_{\text {in }}$ will have one cylindrical end as well as some conical ends $E_{i}, i=1, \ldots, k$, while $\widehat{X}_{\text {out }}$ will be the same as before. The conical ends can be chopped off and replaced by suitable compact manifolds with boundary $Z_{i}$ (as we did before with $\widehat{X}_{\text {out }}$ ) without changing the expected dimension of the corresponding moduli spaces. Then, denoting $\left(\widehat{X}_{\text {in }} \backslash \cup E_{i}\right) \cup Z_{i}$ by $\widetilde{X}_{\text {in }}$, the statement of lemma 3.1 will still hold with $M_{\eta_{\text {in }}}\left(\widehat{X}_{\text {in }}, \theta_{1}\right)$ replaced by $M_{\eta_{\text {in }}}\left(\widetilde{X}_{\text {in }}, \theta_{1}\right)$, and will have a similar proof. On the other hand, the same arguments as before show that, for generic choices of $\eta_{\text {in }}$, the expected dimensions of $M_{\eta_{\text {in }}}\left(\widehat{X}_{\text {in }}, \xi_{1}, \ldots, \xi_{k}, \theta_{1}\right)$ (with the 
obvious meaning of the symbols) and $M_{\eta_{\text {out }}}\left(\widehat{X}_{\text {out }}, \theta_{2}, \xi\right)$ are non-negative, and they coincide with the expected dimensions of $M_{\eta_{\text {in }}}\left(\widetilde{X}_{\text {in }}, \theta_{1}\right)$ and $M_{\eta^{\prime}}\left(\widehat{Z}, \theta_{2}\right)$, respectively. No assumption on $b_{2}^{+}(X)$ is needed, because both $\widehat{X}_{\text {in }}$ and $\widehat{X}_{\text {out }}$ have at least one conical end, and the elements of $M_{\eta_{\text {in }}}\left(\widehat{X}_{\text {in }}, \xi_{1}, \ldots, \xi_{k}, \theta_{1}\right)$ and $M_{\eta_{\text {out }}}\left(\widehat{X}_{\text {out }}, \xi, \theta_{2}\right)$ are asymptotically irreducible on the conical ends. This gives a contradiction as in the previous case, and concludes the proof of the theorem.

Proof of theorem 1.4 Let $\omega$ be the compatible symplectic form. We know (see section 2) that there is a distinguished element $\mathbf{s}_{\omega} \in \operatorname{Spin}^{c}(X, \xi)$ such that $\mathrm{SW}_{(X, \xi)}\left(\mathbf{s}_{\omega}\right) \neq 0$. The conclusion follows immediately from theorem 3.2.

\section{References}

[1] M F Atiyah, V K Patodi, I M Singer, Spectral asymmetry and Riemannian geometry: I, Math. Proc. Cambridge Philos. Soc. 77 (1975) 43-69

[2] D Bennequin, Entrelacements et equations de Pfaff, Astérisque 107-108 (1983), 83-161

[3] S K Donaldson, Connections, cohomology and the intersection forms of fourmanifolds, Jour. Diff. Geom. 24 (1986) 275-341

[4] S K Donaldson, The Seiberg-Witten equations and 4-manifold topology, Bull. AMS 33 (1996) 45-70

[5] Y Eliashberg, Topological characterization of Stein manifolds of dimension $>2$, Intern. Journal of Math. 1, No. 1 (1990) 29-46

[6] Y Eliashberg, Filling by holomorphic discs and its applications, London Math. Soc. Lecture Notes Series 151 (1991) 45-67

[7] Y Eliashberg, Contact 3-manifolds twenty years since J. Martinet's work, Ann. Inst. Fourier 42 (1992) 165-192

[8] Y Eliashberg, Unique holomorphically fillable contact structure on the 3-torus, Intern. Math. Res. Not. 2 (1996) 77-82

[9] N Elkies, A characterization of the $\mathbb{Z}^{n}$ lattice, Math. Res. Lett. 2 (1995) 321326

[10] N Elkies, Lattices and codes with long shadows, Math. Res. Lett. 2 (1995) 643-652

[11] N Elkies, personal communication

[12] J B Etnyre, Symplectic convexity in low dimensional topology, Top. Appl. (to appear) 
[13] K A Frøyshov, The Seiberg-Witten equations and four-manifolds with boundary, Math. Res. Lett. 3 (1996) no. 3, 373-390

[14] E Giroux, Topologie de contact en dimension 3, Séminaire Bourbaki 760 (199293), 7-33

[15] R E Gompf, Handlebody construction of Stein surfaces, Ann. of Math. (to appear)

[16] R Kirby, Problems in Low-Dimensional Topology. In W H Kazez (Ed.), Geometric Topology, Proc of the 1993 Georgia International Topology Conference, AMS/IP Studies in Advanced Mathematics, pp. 35-473, AMS \& International Press (1997)

[17] P B Kronheimer, T S Mrowka, Monopoles and contact structures, Invent. Math. 130 (1997) 209-256

[18] D Kotschick, J W Morgan, C H Taubes, Four-manifolds without symplectic structures but with non-trivial Seiberg-Witten invariants, Math. Res. Lett. 2 (1995) 119-124

[19] F Laudenbach, Orbites périodiques et courbes pseudo-holomorphes, application à la conjecture de Weinstein en dimension 3 [d'après H. Hofer et al.], Astérisque 227 (1995) 309-333

[20] P Lisca, G Matić, Tight contact structures and Seiberg-Witten invariants, Invent. math. 129 (1997) 509-525

[21] J Martinet, Formes de contact sur les variètès de dimension 3, Lect. Notes in Math. 209, Springer-Verlag (1971) 142-163

[22] J Milnor, D Husemoller, Symmetric bilinear forms, Ergebnisse der Mathematik und Ihrer Grenzgebiete, Band 73, Springer-Verlag (1973)

[23] J W Morgan, T S Mrowka, D Ruberman, The $L^{2}-$ moduli space and a vanishing theorem for Donaldson polynomial invariants, Monographs in Geometry and Topology, no. II, International Press, Cambridge, MA, 1994

[24] J W Morgan, T S Mrowka, Z Szabó, Product formulas along $T^{3}$ for SeibergWitten invariants, preprint (1997)

[25] C H Taubes, The Seiberg-Witten invariants and symplectic forms, Math. Res. Lett. 1 (1995) 809-822

[26] C H Taubes, More constraints on symplectic manifolds from Seiberg-Witten equations, Math. Res. Lett. 2 (1995) 9-14 